\title{
Особенности реализации трибодатчиков на основе пьезотрансформаторных измерительных преобразователей с двумя степенями свободы
}

\author{
В.В. Белозерских, В.Н. Седалищев, Я.С. Сергеева
}

Алтайский государственный университет (Барнаул, Россия)

\section{Implementation Features of Tribological Sensors Based on Piezo-Transformer Measuring Converters with Two Degrees of Freedom}

\author{
V.V. Belozerskikh, V.N.Sedalischev, Ya.S. Sergeeva
}

Altai State University (Barnaul, Russia)

\begin{abstract}
Рассмотрены особенности создания трибодатчиков на основе связанных колебаний. Представлена зависимость отношения выходных напряжений пьезоэлектрических трансформаторов от величины возбуждающего напряжения на первой и второй НЧС при разных усилиях сдавливания контакта. Рассмотрены особенности реализации механизма трибочувствительности в датчиках на основе пьезотрансформаторных измерительных преобразователей с двумя степенями свободы. Показано, что, измеряя параметры связанных колебаний контактирующих пьезоэлектрических трансформаторов, можно исследовать физико-механические характеристики контакта. Регулируя амплитуду колебаний пьезорезонаторов и, соответственно, амплитуду взаимных микроперемещений контактирующих поверхностей, устанавливая определенные значения усилий их прижатия, можно реализовать требуемые режимы работы пьезотрансформаторных трибодатчиков. Использование такого механизма трибочувствительности позволит определять твердость материала, шероховатость поверхности, модули упругости продольных и сдвиговых деформаций, коэффициенты трения качения, скольжения, покоя и т.п.
\end{abstract}

Ключевые слова: связанные колебания, пьезорезонансные датчики, система с двумя степенями свободы, MicroCap.

\section{DOI 10.14258/izvasu(2019)4-03}

В настоящее время наблюдается рост числа и разнообразия датчиков, используемых в различных отраслях промышленности, сельском хозяйстве, строительстве и т.п., повышаются требования к качеству измерительного процесса, надежности функционирования средств измерения и контроля, расширению области применения и условий их эксплуатации. В том
The paper discusses the aspects of the creation of tribological sensors based on coupled vibrations. The dependence of the ratio of the output voltages of piezoelectric transformers on the magnitude of the exciting voltage at the first and second normal synchronization frequencies with different amount of exerted pressure is presented. Peculiarities of trisensitivity mechanism implementation in sensors based on piezo-transformer measuring transducers with two degrees of freedom are considered. It is shown that physicomechanical properties of the contact area can be examined by measuring the parameters of coupled oscillations of the contacting piezoelectric resonators. Also, it is possible to set the required operating modes of the piezoelectric resonators of tribological sensors by adjusting the amplitude of oscillation of the piezoelectric resonators and, respectfully, the amplitude of mutual micro-movements of contacting surfaces. The proposed method of measuring the sensitivity is suitable for estimation of hardness and surface roughness of materials, the elastic moduli of longitudinal and shear deformations, the coefficients of rolling friction, slip, and rest, etc.

Key words: coupled oscillation, piezoelectric resonator sensor, two degrees of freedom system, MicroCap. числе исследование физико-механических характеристик поверхностей твердых тел, пленочных покрытий, исследование нано- и микропокрытий в настоящее время является актуальной задачей. Для материалов, обладающих высоким значением упругого восстановления, невозможно корректно определить твердость, используя традиционные методы измерения 
параметров отпечатка. Поэтому традиционные методы определения физико-механических характеристик материалов не всегда применимы для контроля твердых и сверхтвердых покрытий. Это связано с тем, что при малых нагрузках размер отпечатка настолько мал, что его часто невозможно наблюдать в оптический микроскоп, причем большие нагрузки вызывают образование трещин. В связи с этим представляет интерес поиск новых методов определения физикомеханических характеристик поверхностей твердых тел и покрытий для более точной оценки их трибологических свойств [1].

С другой стороны, в настоящее время механизм трибочувствительности не нашел широкого применения в измерительной технике. Низкая стабильность физических процессов, протекающих при трении взаимно перемещающихся поверхностей, не позволяет создавать трибодатчики с высокими метрологическими характеристиками [2]. Поэтому для повышения точности соответствующих средств измерений требуется обеспечить стабильность во времени параметров контактирующих поверхностей. Достигнуть такого состояния можно за счет применения специальных износоустойчивых материалов и покрытий.

Другим недостатком средств измерений, реализующих механизм трибочувствительности, является то, что в существующих в настоящее время трибометрах для создания механических перемещений чаще всего используют электродвигатели. С их помощью обеспечивают взаимное вращательное или поступательное перемещение контактирующих поверхностей. В связи с этим трибодатчики такого типа позволяют измерять только коэффициент трения движения, в то время как с помощью трибометров вибрационного типа можно, изменяя амплитуды возвратно-поступательных перемещений контактирующих поверхностей, исследовать упругие деформации микронеровностей, процессы трения трогания и т.п.

В свою очередь, и вибрационный метод также не лишен определенных недостатков. Например, величина хода должна соответствовать геометрическим размерам неровностей контактирующих поверхностей. Одним из возможных подходов к решению данной проблемы может служить разработка устройства с использованием связанных колебаний пьезотрансформаторов (ПТ), способного осуществлять контроль фактической площади контакта между взаимно перемещающимися поверхностями. Суть предлагаемого метода контроля трибологических характеристик материалов заключается в использовании высокочувствительных режимов связанных колебаний составных пьезотрансформаторов.

На рисунке 1 представлена схема измерительного устройства с фиксированным усилием прижатия элемента связи к исследуемой поверхности (трибодатчик с внешней связью) [3].

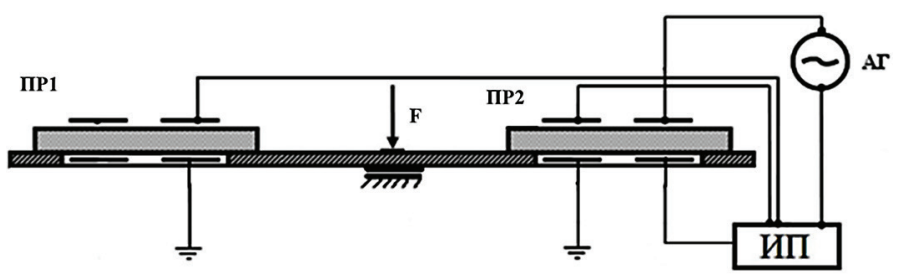

a)

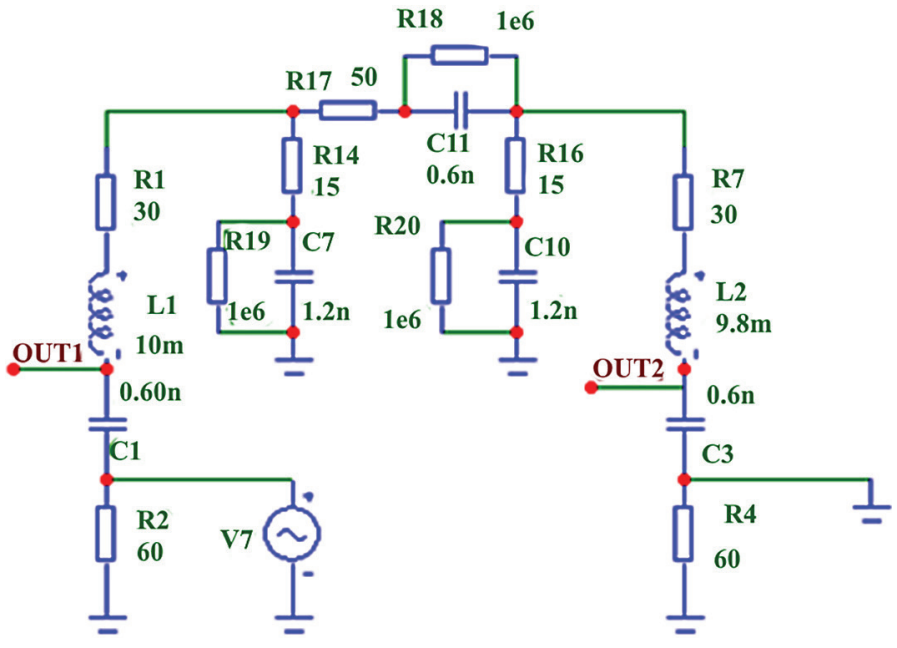

б)

Рис. 1. Структурная схема трибодатчика с внешней связью между составными пьезорезонаторами (a) и его эквивалентная электрическая схема замещения (ЭЭСЗ) (б) 
На рисунке 2 показана структурная схема трибодатчика параметрического типа (трибодатчик с внутренней связью), представляющего собой ко- лебательную систему, состоящую из двух контактирующих между собой составных пьезотрансформаторов.

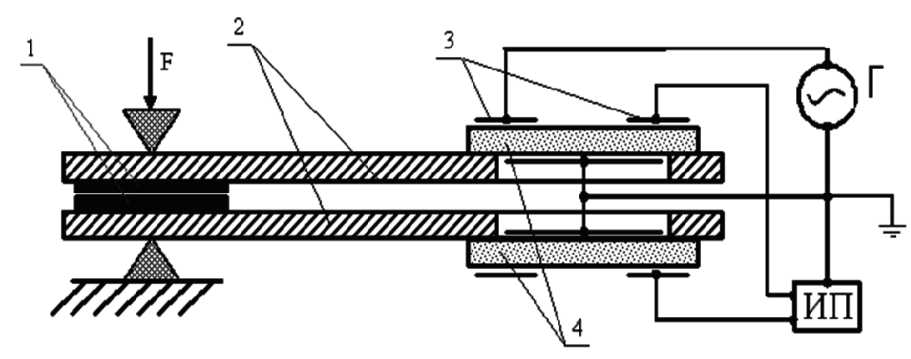

a)

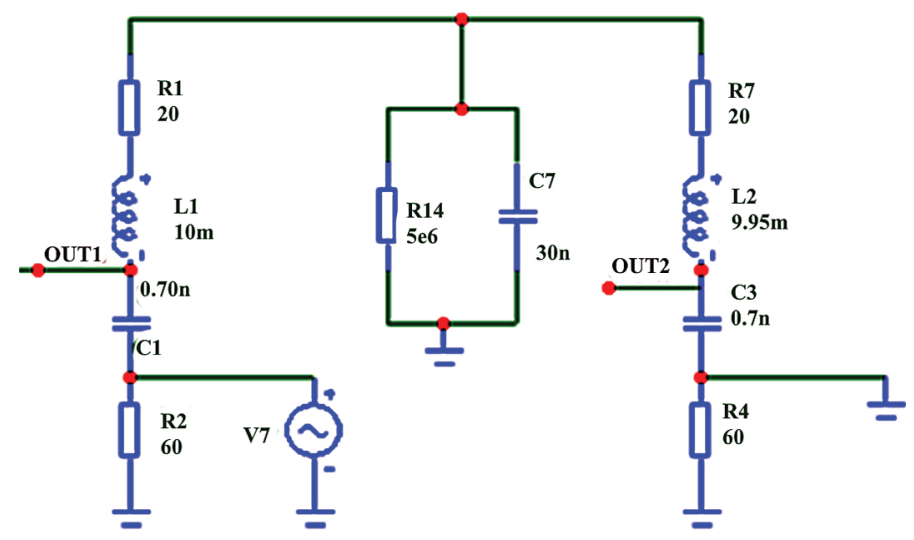

б)

Рис. 2. Структурная схема трибодатчика с внутренней связью между составными пьезорезонансными трансформаторами (а) и его ЭЭСЗ (б)

Трибодатчики вибрационного типа с такой конструкцией чувствительного элемента могут найти применение для измерения различных физических величин: усилий, давлений, уровня жидких и сыпучих сред, микроперемещений, влажности газообразных сред, температуры и др. Порядок и принцип работы измерительных устройств данного типа основан на использовании высокочувствительных процессов связанных колебаний в системах с двумя степенями свободы. В основе механизма чувствительности таких вибрационных трибодатчиков лежит управление процессами трения и упругих взаимодействий между контактирующими поверхностями вибраторов.

Необходимо отметить, что при разработке и проектировании трибодатчиков вибрационного типа необходимо принимать дополнительные меры по оптимизации их конструкции и режимов работы [4]. В частности, с целью повышения метрологических характеристик нужно обеспечивать согласование значений коэффициентов связи и относительной расстройки собственных частот резонаторов с их добротностью. При возбуждении связанных колебаний в системах с двумя степенями свободы связь между резонаторами может быть слабой и сильной. Коэффициент связи определяется упругими свойствами и характером взаимных перемещений контактирующих поверхностей. При увеличении усилия сдавливания контактирующих поверхностей связь между резонаторами возрастает из-за увеличения фактической площади акустического контакта между вибраторами. В результате этого будет возрастать амплитуда колебаний ведомого пьезоэлектрического трансформатора (ПЭТ), соответственно возрастет отношение выходных напряжений ведомого и ведущего ПЭТ [5].

На рисунке 3 приведены зависимости отношения выходных напряжений ПЭТ от величины напряжения возбуждения на первой (а) и второй (б) нормальных частотах синхронизации (НЧС) в системе при различных значениях усилия сдавливания контакта [6]. 


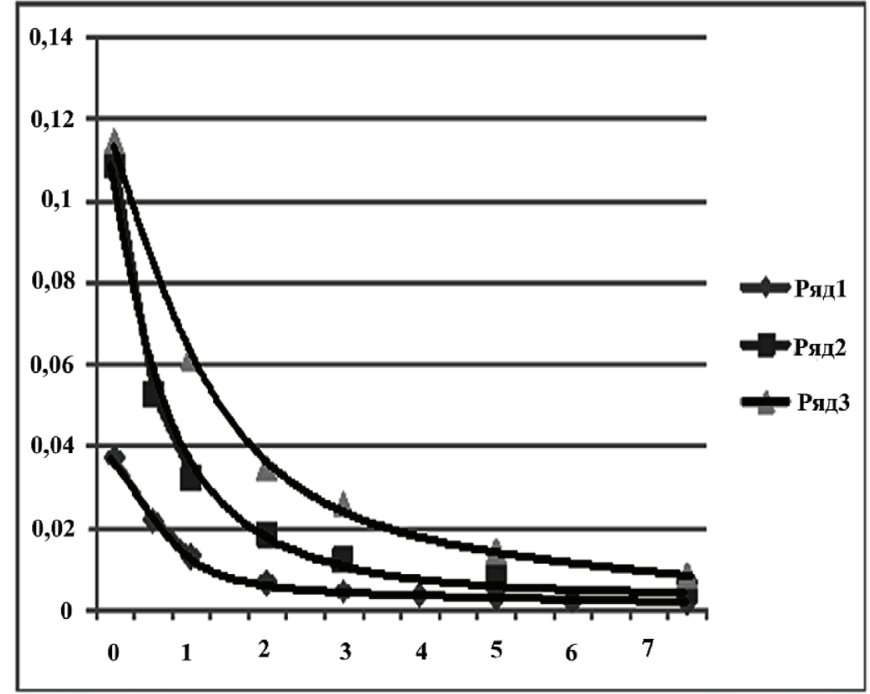

a)

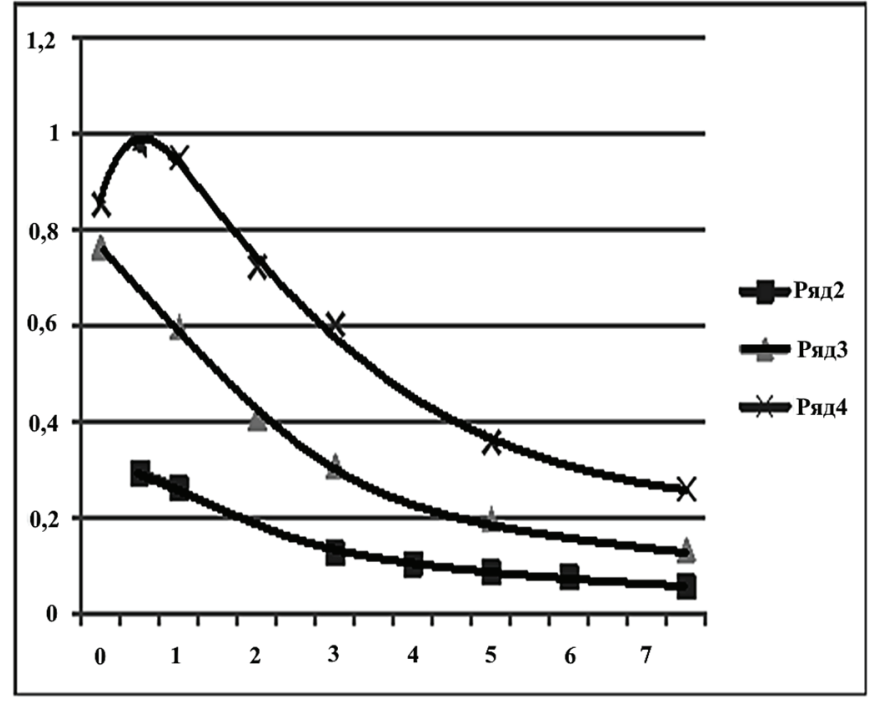

б)

Рис. 3. Зависимость отношения выходных напряжений ПЭТ от величины напряжения возбуждения на первой (а) и второй (б) НЧС при разных усилиях сдавливания контакта

Как следует из приведенных графиков, при увеличении сдавливающих усилий максимум на графике, отражающем зависимость отношения выходных напряжений ПЭТ от уровня напряжения возбуждения, смещается в область больших амплитуд колебаний резонаторов. Это объясняется тем, что при увеличении амплитуд противоположно направленных перемещений поверхностей вибраторов появляется проскальзывание в месте их контакта [7]. В результате этого возрастают потери на трение в месте контакта, уменьшается доля энергии, приходящая к ведомому ПЭТ со стороны ведущего.

На основании полученных зависимостей можно сделать следующие выводы:
- в качестве информативного параметра трибодатчика для регистрации и контроля проскальзывания между контактирующими поверхностями может быть использовано отношение выходных напряжений ПЭТ на частоте противофазных колебаний в системе;

- с целью повышения чувствительности измерений представляет интерес возможность реализации критического режима бифуркации связанных колебаний в системе при постоянном усилии сдавливания контактирующих поверхностей $[8,9]$;

- измеряя отношение выходных напряжений пьезорезонансных трансформаторов при реализации различных видов взаимных перемещений контактирующих поверхностей вибраторов, можно ис- 
следовать физико-механические и трибологические характеристики материалов;

- в зависимости от физических свойств исследуемых материалов, режимов связанных колебаний в колебательной системе измерительного преобразователя (ИП) связь между резонаторами может быть инерционной, упругой, диссипативной, последовательной и параллельной, продольной и поперечной, внешней и внутренней. Разнообразие видов связей между взаимодействующими резонаторами определяет широкие возможности практического использования вибрационного метода для решения широкого круга практических задач по определению характеристик материалов и покрытий [10];

- к достоинствам конструкции трибодатчика можно отнести то, что выходной сигнал формируется в результате перераспределения колебательной энергии между взаимодействующими пьезорезонаторами, что позволяет существенно уменьшить погрешности, обусловленные нестабильностью исполь- зуемых пьезоматериалов, повысить чувствительность измерений за счет реализации критических режимов связанных колебаний в системе, расширить функциональные возможности и область применения датчиков данного типа [11];

- регулируя амплитуду колебаний пьезорезонаторов и, соответственно, амплитуду взаимных микроперемещений контактирующих поверхностей при определенных значениях усилий их прижатия, можно реализовать требуемые режимы работы трибодатчика [12].

Таким образом, измеряя параметры взаимосвязанных колебаний пьезорезонансных трансформаторов, можно исследовать различные физикомеханические и трибологические характеристики материалов, такие как твердость материала, шероховатость поверхности, значение модулей упругости продольных и сдвиговых деформаций материалов, коэффициенты трения качения, скольжения, покоя твердых тел и др. [13].

\section{Библиографический список:}

1. Рабинович М.И., Трубецков Д.И. Введение в теорию колебаний и волн. М., 1992.

2. Богуш М.В., Мокров Е.А., Панич А.Е. Анализ информативности пьезоэлектрических датчиков давления с помощью обобщенного показателя качества // Измерительная техника. 2008. № 6.

3. Шарапов В.М., Сотула Ж. Пьезоэлектрические преобразователи. Новые технологии проектирования // Электроника: наука, технология, бизнес. 2012. № 5.

4. Котюк А. Ф. Датчики в современных измерениях. М., 2006.

5. Малов В.В. Пьезорезонансные датчики. М, 1989.

6. Федосов С.А., Пешек Л. Определение механических свойств материалов микроиндентированием. Современные зарубежные методики. М., 2004.

7. Гуткин М.Ю., Овидько И.А. Физическая механика деформируемых наноструктур. СПб., 2003. Т. 1.

8. Седалищев В. Н. Измерительные устройства, основанные на реализации связанных колебаний в пьезорезонансных датчиковых структурах // Ползуновский вестник. 2006. № 2.
9. Головнин В.А. Физические основы, методы исследования и практическое применение пьезоматериалов. М., 2013.

10. Седалищев В.Н., Сергеева Я.С., Родионов И.М. Особенности реализации механизма трибочувствительности в пьезотрансформаторных измерительных преобразователях с двумя степенями свободы // Новые материалы и технологии : сб. статей VI Российско-казахстанской молод. научно-технич. конфер. Барнаул, 2018.

11. Olszacki M. Modélisation et Optimisation des Capteurs de Pression piézorésistifs. INSA de Toulouse, 2009.

12. Lee K., Gupta N., Sartain R.B. Development Of An Unattended Ground Sensor Array Using Piezoresistive Sensors // Unattended Ground, Sea, And Air Sensor Technologies And Applications XI: Proceedings of the SPIE, 2009. Vol. 7333. Article ID 733316. doi: 10.1117/12.817276.

13. Cao G., Wang X., Xu Y., Liu S. A Micromachined Piezoresistive Pressure Sensor With A Shield Layer. // Sensors. 2016. Vol. 16, no. 8. 